\title{
Measurement of the Plasma Boundary Shift and Approximation of the Magnetic Surfaces on the IR-T1 Tokamak
}

\author{
A. Salar Elahi \\ Physics Department, Faculty of Engineering, Islamic Azad University, Saveh Branch, Saveh, Iran. \\ M. Ghoranneviss \\ Plasma Physics Research Center, Science and Research Branch, \\ Islamic Azad University, P.O. Box 14665-678, Tehran, Iran
}

(Received on 15 February, 2010)

\begin{abstract}
In this research we measured the plasma boundary shift using array of magnetic pick-up coils on the IR-T1 tokamak. Also we approximated the magnetic surfaces by an equilibrium calculation. Firstly, four magnetic probes were designed, constructed, and installed on outer surface of the IR-T1 tokamak chamber and then plasma boundary displacement measured from them. On the other hand, magnetic surfaces approximated by equilibrium calculation of the Grad-Shafranov equation based on expansion of free functions as quadratic in flux function.
\end{abstract}

Keywords: Tokamak, Plasma Boundary Shift, Magnetic Surfaces.

\section{INTRODUCTION}

To a very good approximation in tokamaks, the problem of achieving toroidal equilibrium separates into two parts. First, the magnetic configuration must provide radial confinement, i.e., radial pressure balance in the poloidal plane so that the pressure contours form closed nested surfaces. Second, the configuration must compensate the radially outward expansion force inherent in all toroidal geometries, i.e., toroidal force balance. But, in the second one, plasma may intend to shift inward or outward, which is very dangerous for tokamak plasma. Therefore, plasma equilibrium study is one of the fundamental problems of the magnetically confined plasmas. There are many available global solutions of the steady state magnetohydrodynamics equations, in particular, the Grad-Shafranov equation. Control of plasma position plays an important role in plasma confinement and to achieve optimized tokamak plasma operation. Accurate determination of the plasma position during confinement time is essential to transport it to a control system based on feedback. Over the years different methods have been developed to analyze the equilibrium problems and determination of both plasma boundary shift and magnetic surfaces [1-21].

In this paper we presented magnetic probes method for measurement of the plasma boundary shift and approximation of magnetic surface based on analytical solution of the Grad-Shafranov equation on the IR-T1 Tokamak, which is a small, air core, low beta and large aspect ratio tokamak with a circular cross section, (see Table 1). Details of the magnetic probes method for the determination of plasma boundary shift will be discussed in section 2. Approximation of magnetic surfaces based on analytical solution of the GradShafranov equation will be presented in section 3. Experimental result also will be presented in section 4. Summary and conclusion are also will be presented in section 5 .

\section{MAGNETIC PROBES TECHNIQUE FOR THE MEASUREMENT OF PLASMA BOUNDARY SHIFT}

Because of dependence of the plasma position and plasma current distribution to the magnetic field distributions around the plasma, magnetic pickup coils can give us information about the plasma position or plasma boundary shift. Poloidal and normal components of the magnetic fields distribution in the quasi-cylindrical coordinates $(r, \theta, \phi)$ around the circular cross section plasma are [1]:

$$
\begin{aligned}
& B_{\theta}=\frac{\mu_{0} I_{p}}{2 \pi b}-\frac{\mu_{0} I_{p}}{4 \pi R_{0}} \times \\
& \left\{\ln \frac{a}{b}+1-\left(\Lambda+\frac{1}{2}\right)\left(\frac{a^{2}}{b^{2}}+1\right)-\frac{2 R_{0} \Delta R}{b^{2}}\right\} \cos \theta, \\
& B_{r}=-\frac{\mu_{0} I_{p}}{4 \pi R_{0}} \times \\
& \left\{\ln \frac{a}{b}+\left(\Lambda+\frac{1}{2}\right)\left(\frac{a^{2}}{b^{2}}-1\right)+\frac{2 R_{0} \Delta R}{b^{2}}\right\} \sin \theta,
\end{aligned}
$$

where $\Lambda=\beta_{p}+l i / 2-1$ is the Shafranov parameter and $a, b$, $I_{p}, R_{0}, \beta_{p}, l i$, and $\Delta R$ are the plasma minor radius, chamber minor radius, plasma current, chamber major radius, poloidal beta, internal inductance, and plasma boundary horizontal shift, respectively. Therefore, by rearranging the above equations the following relation for the horizontal shift of the plasma boundary is obtained:

$$
\begin{aligned}
& \Delta R=\frac{a^{2}}{4 R_{0}}\left\{\left(\frac{b^{2}}{a^{2}}-1\right)-2 \ln \frac{a}{b}\right\}+ \\
& \frac{\pi b^{2}}{2 \mu_{0} I_{p}}\left\{\Delta B_{1 \theta}\left(1-\frac{a^{2}}{b^{2}}\right)-\Delta B_{r}\left(1+\frac{a^{2}}{b^{2}}\right)\right\} .
\end{aligned}
$$

As observable, this relation depends only on the plasma current and magnetic fields distribution. Also the vertical displacement of the plasma boundary is [2]:

$$
\Delta Z=\frac{\pi b^{2}}{\mu_{0} I_{p}} \Delta B_{2 \theta}
$$

Equations (1) and (2) are accurate for the low beta, large aspect ratio, and circular cross section tokamaks as IR-T1, also:

$$
\begin{aligned}
& \Delta B_{1 \theta}=B_{\theta}(\theta=0)-B_{\theta}(\theta=\pi), \\
& \Delta B_{2 \theta}=B_{\theta}(\theta=3 \pi / 2)-B_{\theta}(\theta=\pi / 2), \\
& \Delta B_{r}=B_{r}(\theta=\pi / 2)-B_{r}(\theta=3 \pi / 2) .
\end{aligned}
$$


According to this approach, four magnetic probes were designed, constructed, and installed on the IR-T1 tokamak, two magnetic probes were located on the circular contour $\Gamma$ of the radius $b=16.5 \mathrm{~cm}$ in angles of $\theta=0$ and $\theta=\pi$ to detect the tangential component of the magnetic field $B_{\theta}$ and two magnetic probes are also located above, $\theta=\pi / 2$, and below, $\theta=3 \pi / 2$, to detect the normal component of the magnetic field $B_{r}$ (see the Fig. (1)).

By substituting the poloidal and normal components of the magnetic fields which are measured by the four magnetic pickup coils (after compensation and integration of their output) in Equations (3) and (4) $\left(\Delta B_{2 \theta}\right.$ measured using the poloidal Mirnov coils), horizontal and vertical displacements of the plasma boundary were determined. Experimental results will be presented in the section 4 .

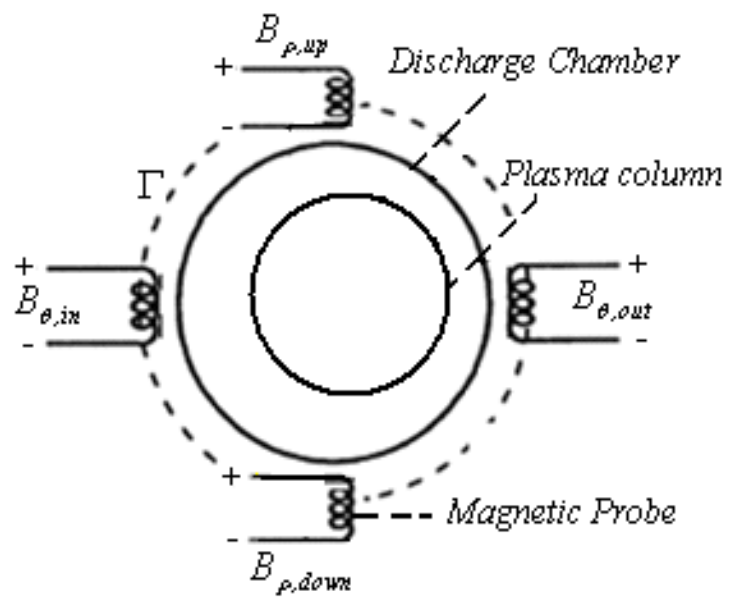

FIG. 1: Positions of the four magnetic probes on outer surface of the IR-T1 tokamak chamber.

\section{APPROXIMATION OF THE MAGNETIC SURFACES BASED ON ANALYTICAL SOLUTION OF THE GRAD-SHAFRANOV EQUATION}

Maxwell's equations together with the force balance equation from MHD equations, in the cylindrical coordinates $(R, \mathrm{Z})$ reduce to the two-dimensional, nonlinear, elliptic partial differential equation, or Grad-Shafranov equation [3]:

$$
\Delta^{*} \psi=-\mu_{0} R^{2} \frac{d p}{d \psi}-F \frac{d F}{d \psi},
$$

where

$$
\begin{gathered}
\vec{B}=\frac{1}{R} \vec{\nabla} \psi \times \hat{e}_{\phi}+\frac{F}{R} \hat{e}_{\phi}, \\
\mu_{0} \vec{J}=\frac{1}{R} \frac{d F}{d \psi} \vec{\nabla} \psi \times \hat{e}_{\phi}-\frac{1}{R} \Delta^{*} \psi \hat{e}_{\phi},
\end{gathered}
$$

and $p(\psi)$ and $F(\psi)$ are two free functions, and where $\mu_{0}$ and $J$ are the vacuum permeability and plasma current density respectively. Many authors solved the Grad-Shafranov equation by expanding the free functions on different order in $\psi$. In this section, we presented quadratic order (which proposed by Guazzotto [4]), and approximated on the IRT1. If we choose the free functions to be quadratic in $\psi$ as [4]:

$$
\begin{gathered}
p=p_{0}\left(\psi^{2} / \psi_{0}^{2}\right), \\
F^{2}=R_{0}^{2} B_{0}^{2}\left[1+b_{0}\left(\psi^{2} / \psi_{0}^{2}\right)\right],
\end{gathered}
$$

where $\psi_{0}, p_{0}, b_{0}$ are the values of $\psi, p$, and $F$ on magnetic surfaces axis, $R_{0}$ is the tokamak major radius, and $B_{0}$ is the vacuum toroidal field.

The Grad-Shafranov equation reduces to:

$$
\Delta^{*} \psi=-\frac{R_{0}^{2} B_{0}^{2}}{\psi_{0}^{2}}\left(b_{0}+\beta_{0} \frac{R^{2}}{R_{0}^{2}}\right) \psi,
$$

where $\beta_{0}=2 \mu_{0} p_{0} / B_{0}^{2}$. With normalizing variables as $R^{2} / R_{0}^{2}=x$, and $Z / a=y$, Eq. (9) can then be written as:

$$
4 \varepsilon^{2} x \frac{\partial^{2} \psi}{\partial x^{2}}+\frac{\partial^{2} \psi}{\partial y^{2}}+(\alpha x+\gamma) \psi=0
$$

where $\varepsilon=a / R_{0}$, is the inverse aspect ratio, and

$$
\alpha=\left(\frac{a R_{0} B_{0}}{\psi_{0}}\right)^{2} \beta_{0}, \gamma=\left(\frac{\mathrm{aR}_{0} B_{0}}{\psi_{0}}\right)^{2} b_{0} .
$$

The solution of Eq. (10) in cylindrical coordinates $(R, \mathrm{Z})$ can be written as:

$$
\psi=\sum_{m} X_{m}(\rho) Y_{m}(y)
$$

where $x=-i(\varepsilon / \sqrt{\alpha}) \rho$, and for up-down symmetric case, $Y_{m}$ obtained as:

$$
\begin{aligned}
& \frac{d^{2} Y_{m}}{d y^{2}}+k_{m}^{2} Y_{m}=0, \\
& Y_{m}(y)=\cos \left(k_{m} y\right) .
\end{aligned}
$$

Also $X_{m}(\rho)$ can be written as:

$$
\begin{aligned}
& \frac{d^{2} X_{m}}{d \rho^{2}}+\left[\frac{\lambda_{m}}{\rho}-\frac{1}{4}\right] X_{m}=0, \\
& X_{m}(\rho)=a_{m} W_{\lambda_{m}, \mu}(\rho)+b_{m} M_{\lambda_{m}, \mu}(\rho),
\end{aligned}
$$

where $\lambda_{m}=-i \frac{\gamma-k_{m}^{2}}{4 \varepsilon \sqrt{\alpha}}, W_{\lambda_{m}, \mu}(\rho)$, and $M_{\lambda_{m}, \mu}(\rho)$ are the Whittaker functions, and in this model $\mu=1 / 2$.

Guazzotto proposed only three terms for $m$, and then $\psi$ can be written as:

$$
\begin{aligned}
& \psi(\rho, y)= \\
& \sum_{m=1}^{3}\left[a_{m} W_{\lambda_{m}, 1 / 2}(\rho)+b_{m} M_{\lambda_{m}, 1 / 2}(\rho)\right] \cos \left(k_{m} y\right)
\end{aligned}
$$

where $a_{m}, b_{m}$, and $k_{m}$ are nine unknown coefficients which must be determined. The first six of them can be obtained 
from boundary conditions. The boundary conditions for the points of inner, outer, and top of the plasma cross section are (see Figure (2)):

$$
\begin{aligned}
& \psi\left(R_{0}+a, 0\right)=0, \quad \psi\left(\mathrm{R}_{0}-a, 0\right)=0, \\
& \psi\left(\mathrm{R}_{0}, \mathrm{a}\right)=0, \frac{\partial \psi}{\partial \mathrm{R}}\left(R_{0}, a\right)=0,
\end{aligned}
$$

where $a$ is the plasma radius, and condition for right convexity on the inboard midplane is:

$$
\frac{1}{R_{c}}=\frac{\frac{\partial^{2} \psi}{\partial Z^{2}}\left(R_{0}-a, 0\right)}{\frac{\partial \psi}{\partial \mathrm{R}}\left(R_{0}-a, 0\right)}=-\frac{1}{a},
$$

also two conditions (one defining the location of the magnetic axis $\left(R_{\text {axis }}\right)$ and the other the normalization for $\psi$ on magnetic axis) are:

$$
\frac{\partial \psi}{\partial R}\left(R_{\text {axis }}, 0\right)=0, \frac{\psi\left(\mathrm{R}_{\mathrm{axis}}, 0\right)}{\psi_{0}}=1 .
$$

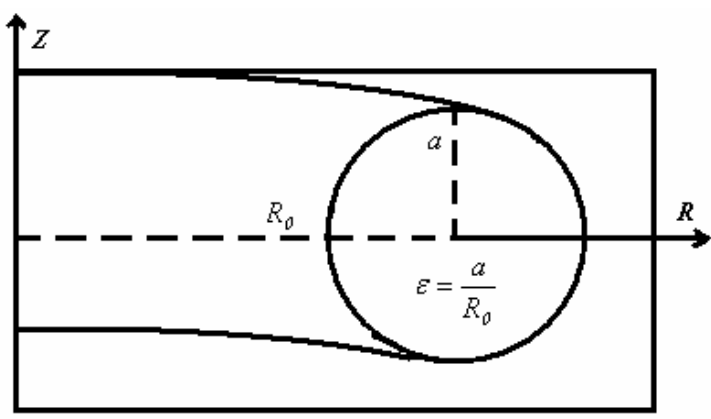

FIG. 2: geometry used for the boundary conditions.

These are seven boundary conditions for seven unknown coefficients $\left(a_{m}, \mathrm{~b}_{\mathrm{m}}, \mathrm{R}_{\mathrm{axis}}\right)$.

But for other three coefficients, by setting $k_{1}=0$ (the simplest solution for GSE independent of $Z$ ), and introduce one approximate value for the $\gamma\left(\gamma \approx 2(q / \varepsilon)^{2}\left(\delta B_{\phi} / B_{\phi}\right)\right.$, negative for diamagnetism plasma, and where $q=a B_{\phi} / R_{0} B_{\theta}$ and $\varepsilon$ are the safety factor and inverse aspect ratio respectively), and assuming that $k_{2}$ be imaginary and $k_{3}$ be real, the values of $\alpha, \mathrm{k}_{2}, \mathrm{k}_{3}$ can be approximated by minimizing the error function between traditional plasma shape $(R=$ $R_{0}+a \cos \theta, Z=a \sin \theta$ ), and analytical plasma shape. Appropriate error function between them defined as follow [4]:

$$
\begin{aligned}
& \text { Error Function }= \\
& \frac{\sum_{i} \sqrt{\left[R_{A}\left(\theta_{i}\right)-R_{T}\left(\theta_{i}\right)\right]^{2}+\left[Z_{A}\left(\theta_{i}\right)-Z_{T}\left(\theta_{i}\right)\right]^{2}}}{\sum_{i} \sqrt{R_{T}^{2}\left(\theta_{i}\right)+Z_{T}^{2}\left(\theta_{i}\right)}},
\end{aligned}
$$

where subscripts ( $A$ and $T$ ) indicates the analytical and traditional plasma shape parameters respectively, and sum in minimum include 3 angles $(0, \pi / 2, \pi)$ for our purpose (circular plasma).

In general by minimizing the error function (e.g. by Mathematica software) as possible to zero, and finding optimal values for $\alpha, k_{2}, k_{3}$, and also solving seven equations for the boundary conditions (Eqs. (16), (17), and (18), six unknown coefficients $\left(a_{m}, \mathrm{~b}_{\mathrm{m}}\right)$, moreover $R_{\text {axis }}$ can be find. Therefore the approximated magnetic surfaces can be plotted by substituting these nine coefficients and also input parameters as $I_{p}$, $\alpha, R_{0}$, and $\varepsilon$ in Eq. (15). For example the magnetic surfaces at $t=15 \mathrm{~ms}$ correspond to approximated $\gamma \approx-0.32$ be shown in the Fig. (5).

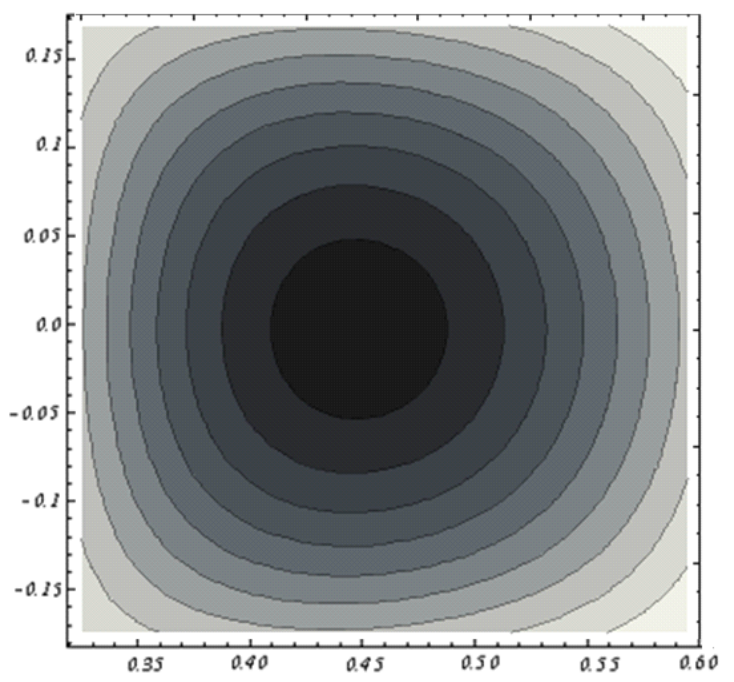

FIG. 3: Magnetic Surfaces Approximated by the Analytical Method at $\mathrm{t}=15 \mathrm{~ms}$ in Target Shot correspond to $\gamma \approx-0.32$ on IR-T1 Tokamak, Displacement of the magnetic surfaces relatively to the plasma boundary observable.

\section{EXPERIMENTAL RESULT}

For the determination of plasma boundary shift using the magnetic probes method, we needed for determination of the magnetic fields distribution around the plasma. Therefore we designed, and constructed a four magnetic pickup coils, and installed them on outer surface of the IR-T1 chamber, and then desired components of the magnetic fields were measured. From this measurement and using the Eq. (3), the plasma boundary shift measured. The Figure (4) shows the result. 


\begin{tabular}{|c|c|}
\hline Parameters & Value \\
\hline Major Radius & $45 \mathrm{~cm}$ \\
\hline Minor Radius & $12.5 \mathrm{~cm}$ \\
\hline Toroidal Field & $\langle 1.0 \mathrm{~T}$ \\
\hline Plasma Current & $\langle 40 \mathrm{kA}$ \\
\hline Discharge Time & $\langle 35 \mathrm{~ms}$ \\
\hline Electron Density & $0.7-1.5 \times 10^{13} \mathrm{~cm}^{-3}$ \\
\hline
\end{tabular}

Table 1. Parameters of the IR-T1 Tokamak
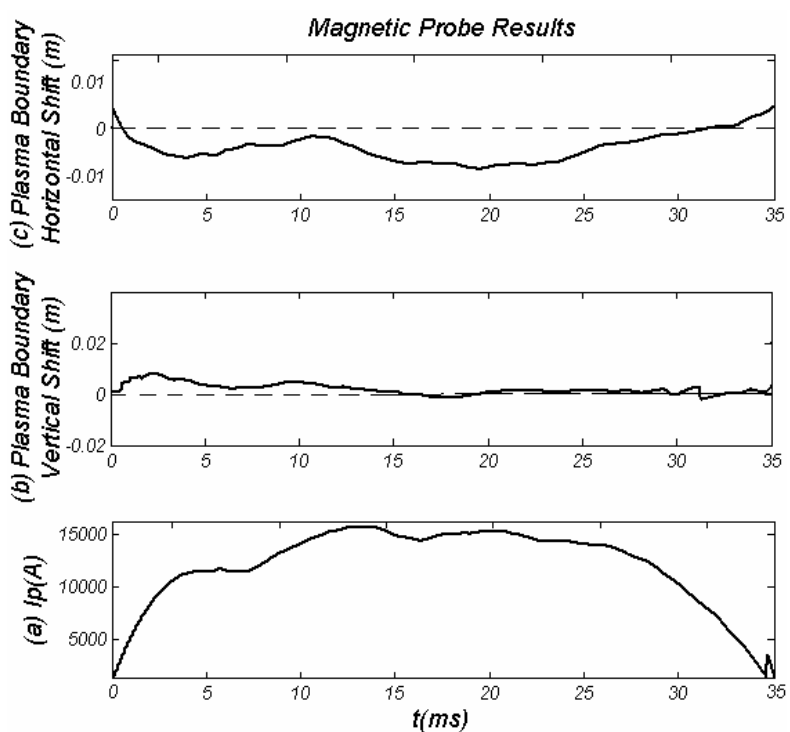

FIG. 4: (a) Plasma current, (b) Time interval of the Plasma Boundary Vertical Displacement, and (c) Time Interval of the Plasma Boundary Horizontal Displacement measured by the magnetic probes.

\section{SUMMARY AND CONCLUSION}

In this research we measured the plasma boundary shift using array of magnetic pick-up coils on the IR-T1 tokamak. Also we approximated the magnetic surfaces by an equilibrium calculation. Firstly, four magnetic probes were designed, constructed, and installed on outer surface of the IR-T1 tokamak chamber and then plasma boundary displacement determined from them. The possible minor errors in this research are because of (1) approximation in the measurement of magnetic fields distribution because of discrete probes measurement, (2) possible error in the compensation of error fields, (3) the approximate values chosen for $\gamma$, and (4) the errors do not become zero during minimizing the error function.
[1] H. Niomiya and N. Suzuki, Jpn. J. Applied Phys., 21 (9), 1323 (1982).

[2] M. Valovic, Czech. J. Phys., B38, 65 (1988).

[3] J. P. Freidberg, Ideal MHD (Clarendon, Oxford, 1987).

[4] L. Guazzotto and J. P. Freidberg, PSFC/JA-07-14, Phys. Plasmas, (2007).

[5] C. V. Atanasiu et al., Phys. Plasmas, 11, 3510 (2004).

[6] G. S. Lee et al., Nucl. Fusion 41, 1515 (2001).

[7] S. H. Seo, Phys. Plasmas 16, 032501 (2009).

[8] S. G. Lee et al., Rev. Sci. Instrum. 79, 10F117 (2008).

[9] A. J. H. Donne, Nucl. Fusion 47, S337-S384, (2007).

[10] F. Najmabadi et al., Fusion Eng. Des. 65, 143 (2003).

[11] L. Guazzotto et al., Phys. Plasmas 11, 604 (2004).
[12] A. Salar Elahi et al., IEEE Trans. Plasma Science 38 (2), (2010).

[13] A. Salar Elahi et al., J. Phys. Scripta 80, 045501 (2009).

[14] A. Salar Elahi et al., J. Phys. Scripta 80, 055502 (2009).

[15] M. Emami et al., J. Plasma Physics 76 (1), (2009).

[16] A. Salar Elahi et al., J. Fusion Energy 28 (4), 346 (2009).

[17] A. Salar Elahi et al., J. Fusion Energy 28 (4), 390 (2009).

[18] A. Salar Elahi et al., J. Fusion Energy 29 (1), 83 (2010).

[19] A. Salar Elahi et al., J. Fusion Energy 29 (1), 88 (2010).

[20] A. Rahimi Rad et al., J. Fusion Energy 29 (1), 73 (2010).

[21] A. Salar Elahi et al., J. Phys. Scripta 81 (5), 055501 (2010). 